\title{
Development of a Low-Volume Sprayer for an Unmanned Helicopter
}

\author{
Yanbo Huang ${ }^{1}$, W. Clint Hoffman ${ }^{2}$, Yubin Lan ${ }^{3}$, Bradley K. Fritz ${ }^{2} \&$ Steven J. Thomson ${ }^{1}$ \\ ${ }^{1}$ United States Department of Agriculture, Agricultural Research Service, Crop Production Systems Research \\ Unit, Stoneville, Mississippi, USA \\ ${ }^{2}$ United States Department of Agriculture, Agricultural Research Service, Aerial Application Technology \\ Research Unit, College Station, Texas, USA \\ ${ }^{3}$ South China Agricultural University, College of Engineering, Guangzhou, China \\ Correspondence: Yanbo Huang, USDA-ARS, Crop Production Systems Research Unit, 141 Experiment Station \\ Road, Stoneville, Mississippi 38776, USA. E-mail: yanbo.huang@ars.usda.gov
}

\author{
Received: October 23, 2014 Accepted: November 18, 2014 Online Published: December 15, 2014 \\ doi:10.5539/jas.v7n1p148 URL: http://dx.doi.org/10.5539/jas.v7n1p148
}

\begin{abstract}
An UAV (Unmanned Aerial Vehicle) can target much smaller areas with lower flight altitudes than conventional, piloted airplanes. In agriculture, UAVs have been mainly developed and used for chemical application and remote sensing. Application of fertilizers and chemicals is frequently needed at specific time and location for highly accurate site-specific management. UAVs provide a technology to fulfill the goals of such site-specific crop management as part of modern precision applications to agricultural crops. This research focused on development of a new low-volume sprayer for an UAV helicopter to be used for vector control, which is potentially extended to crop production management. The developed UAV-based low-volume sprayer was able to deliver liquid covering the $30-\mathrm{m}$ swath width, 42-m downwind. Deposition results from monofilament lines demonstrated that the spray coverage was sensitive to the power voltage but not release height, while the deposition measurement from rotary impactors may need to be refined to achieve higher resolution and lower variance. The developed UAV application technology is useful for precision delivery of chemicals to the right place at right time.
\end{abstract}

Keywords: unmanned aerial vehicle, unmanned helicopter, chemical spray

\section{Introduction}

An unmanned aerial vehicle (UAV) is an unpiloted unmanned aircraft that can be remotely controlled or flown autonomously based on pre-programmed flight plans or more complex dynamic automated guidance systems. UAVs have been developed to support the practice of precision agriculture (Huang et al., 2013). Piloted aircraft that carry a regular sprayer can cover well over hundreds of hectares of crop fields. However, piloted aircraft are not prevalent in all areas, so UAV alternatives are needed. Field operations over smaller fields can especially benefit from use of UAVs.

The Yamaha Motor Corporation (Iwata, Shizuoka, Japan) produces the most advanced non-military UAV (Sato, 2003). Primarily the Yamaha UAVs were developed and used for agriculture application, like insect pest control of rice paddies, soybeans and wheat. The first system, RCASS (Remote-Controlled Aerial Spraying System), was built in 1980. Later on, in 1990, the helicopter R50 had a payload of $20 \mathrm{~kg}$ and a laser-system for height determination. In 1997, the RMAX model was equipped with an azimuth and differential Global Positioning System (GPS) sensor system (Sato, 2003). Miller (2005) reported an experiment to determine the effectiveness of using an UAV for dispersing pesticides to reduce human disease due to insects. In the experiment a commercial off-the-shelf Yamaha UAV, the RMAX type, was outfitted with both liquid and granular pesticide dispersal devices, and a series of tests were performed to evaluate the effectiveness of the UAV to perform aerial pesticide delivery.

Huang et al. (2009) developed a low volume spray system in the laboratory for use on an unmanned vertical take-off and landing helicopter (Rotomotion, LLC, Charleston, South Carolina, USA). The Huang et al. (2009) spray system was developed and integrated with the flight control system of the helicopter, which has a maximum payload of $22.7 \mathrm{~kg}$. The integrated sprayer was developed to be triggered by preset positional coordinates received from the GPS system of the UAV through a PWM (Pulse Width Modulation) pump speed 
controller. The research from this paper further developed and evaluated the system for low volume spray in a small scale field, which will promote the application of the system for pesticide application for vector control from human diseases and crop production management in precision agriculture.

\section{System Design}

\subsection{UAV Payload Configuration}

The low-volume sprayer was designed for the Rotomotion's UAV helicopter. The helicopter has a main rotor diameter of $3 \mathrm{~m}$ and a maximum payload of $22.7 \mathrm{~kg}$. After subtracting for the $2.25 \mathrm{~kg}$ standard undercarriage, the net useable payload is $20.25 \mathrm{~kg}$. If a $0.45 \mathrm{~kg}$ generator, a $0.45 \mathrm{~kg}$ high-performance telemetry, and a gallon of gas with tank $(3.15 \mathrm{~kg})$ are deducted, $15.75 \mathrm{~kg}$ payload is left for mechanical and electronic components of the sprayer. These components include spray pump, pump speed controller, chemical with tank, tubing, and nozzles.

\subsection{Sprayer Design and Development}

In designing the sprayer for the UAV, an application rate of $0.3 \mathrm{~L} / \mathrm{ha}$ was desired covering at least 14 ha per spray mission. The specific gravity of the chemical used in this study was $3.3 \mathrm{~kg} / \mathrm{gal}$; therefore, $5.7 \mathrm{~L}$ ( $1.5 \mathrm{gal}$ ) chemical was needed to cover the 14 ha land. If the spray swath width is 30 meters swath and the air speed of 2.2 $\mathrm{m} / \mathrm{s}(5 \mathrm{mph})$, the pumping rate of the sprayer needs about $100 \mathrm{~mL} / \mathrm{min}$. The helicopter uses one gallon of gas for every 45 minutes.

The low-volume sprayer was constructed to be mounted onto the UAV helicopter. The sprayer was directly interfaced with UAV's electronic control systems to trigger spray release based on specified GPS coordinates and preprogrammed spray locations. The sprayer mainly includes four components: a boom arm with mounted spray nozzles; a tank to house the spray material; a liquid gear pump; and amechanism electronic box to control spray activation. All of these components, along with chemical, must weigh less than $15.75 \mathrm{~kg}$ as discussed in section 2.1 .

Micronair ULV-A+ rotary nozzles (Micron Sprayers Ltd, Bromyard, Herefordshire, UK) were selected for the sprayer which generated a spray volume mean diameter (VMD, referred to the diameter $50 \%$ of the spray volume is made up of droplets less than and greater than) less than $50 \mu \mathrm{m}$ (Huang et al., 2009). A $5.7 \mathrm{~L}$ (1.5 gal) spray tank was designed and built with two internal baffle plates to minimize sloshing of the spray material load during flight (Huang et al., 2009). The tank weighed $1 \mathrm{~kg}$ plus $5 \mathrm{~kg}$ for $5.7 \mathrm{~L}$ of chemical, which resulted in the $9.75 \mathrm{~kg}$ net payload of the UAV.

Aluminum tubing with all-plastic Micronair nozzles, gear pump, and control box restricted the weight of the rest of components of the sprayer to less than $9.75 \mathrm{~kg}$. Figure 1 shows the low-volume sprayer integrated with the UAV helicopter.

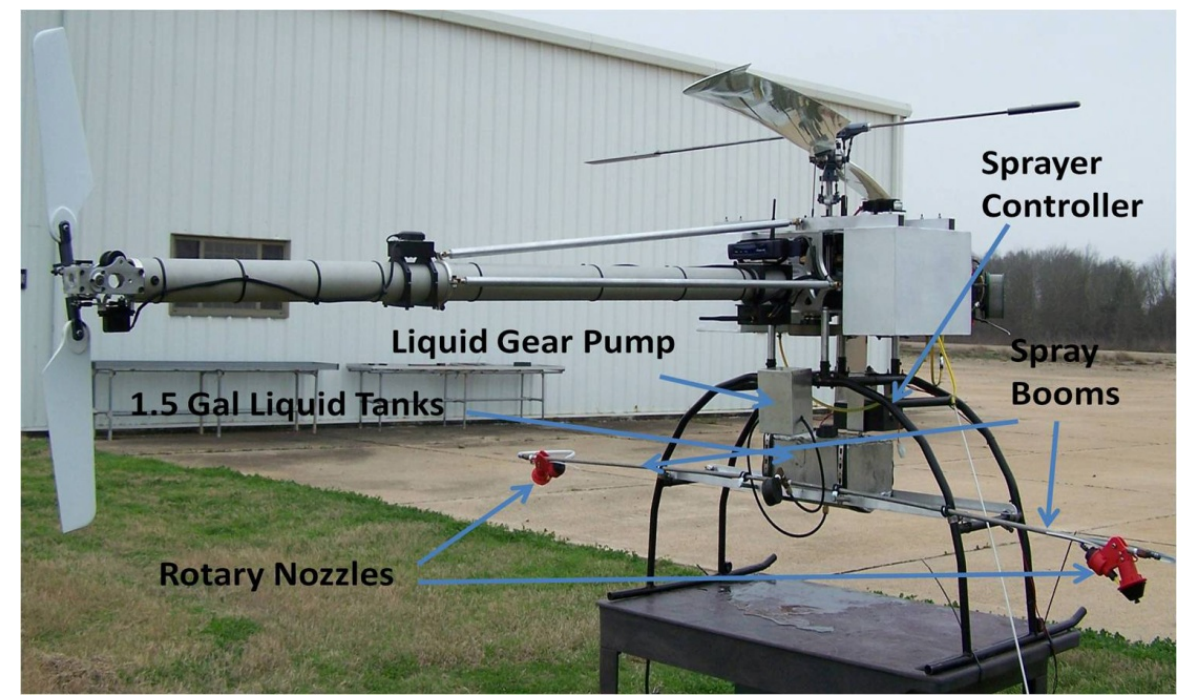

Figure 1. UAV helicopter spray system with spray system mounted on the undercarriage (modified from Huang et al., 2013) 


\section{Sprayer Field Evaluation}

\subsection{Lab Evaluation}

The low-volume sprayer for the UAV was evaluated by spraying BVA oil, a crop oil used to mimic real-world tank solutions, thereby, limiting the use of active ingredients in nozzle test (Huang et al., 2009). The evaluation was conducted using the measured flow rates to determine the needed number of nozzles needed on the spray system for the 30-m swath width, air speed of $2.2 \mathrm{~m} / \mathrm{s}$, and a spray rate of $0.3 \mathrm{~L} / \mathrm{ha}$. The results indicate that with $50 \mu \mathrm{m}$ or less VMD droplet size for vector control for the targeted spray rate, two, three and four Micronair ULV-A+ rotary nozzles are needed, depending on the applied pump voltage (Huang et al., 2009).

\subsection{Field Evaluation Configuration}

A field test was conducted on August 27, 2008 in College Station, Texas to determine the sprayed deposition in a 30 -m swath width of the low-volume sprayer. In the test, the sprayer was attached on the top of a pneumatic controlled variable-height pole and mounted on a heavy-duty truck to mimic the operation of the sprayer with the flight speed and height of the helicopter. Three Micronair ULV A+ rotary nozzles were used for the test on the boom. The nozzles were calibrated to deliver $100 \mathrm{~mL} / \mathrm{min}$. The sprayer released the liquid at the heights of $3.7 \mathrm{~m}$ and $6.1 \mathrm{~m}$ driven at $4 \mathrm{~V}$ and $6 \mathrm{~V} \mathrm{DC}$ (Direct Current) power for less than $50 \mathrm{~m}$ VMD droplet size. The moving speed of the spray structure was at $2.2 \mathrm{~m} / \mathrm{s}$. The spray solution was the mixture of the BVA 13 ULV Oil ${ }^{\circledR}$ (Adapco, Inc., Sanford, FL), which has very similar physical properties to the oil-based insecticide Anvil $10+10 \AA$ (Clarke Mosquito Control, Roselle, Illinois) and Uvitex, a fluorescent dye, at $1.25 \mathrm{~g} / \mathrm{L}$ for spectrofluorometer readings related to spray deposition.

\subsection{Site Layout for Field Evaluation}

Two spray measuring devices were used: monofilament lines and hock rotary impactors (Fritz et al., 2011). The monofilament lines $\left(0.457 \mathrm{~mm}\right.$ diameter line by $30 \mathrm{~m}$ length for total collection area of $\left.137 \mathrm{~cm}^{2}\right)$ and rotary impactors $(2,7 \times 250 \mathrm{~cm}$ acryl glass slides held $16 \mathrm{~cm}$ apart and rotated at a speed of $3.6 \mathrm{~m} / \mathrm{s}$ for a total collection area of $112 \mathrm{~cm}^{2}$ ) were used to capture airborne sprayed materials at distances from $0-42 \mathrm{~m}$ downwind of the spray line. The rotary impactors were used to capture fine droplets floating in the air in the swath downwind. Figure 2 shows the layout the test field. In the field monofilament lines were placed at $0,6,12,18$, $24,30,36$ and $42 \mathrm{~m}$ downwind from spray structure moving line, $30 \mathrm{~m}$ sections of monofilament lines were held at $1 \mathrm{~m}$ above the ground. The lines were collected onto specially constructed holders using cordless drills. The spray lines were placed in a labeled holder. The rotary impactors (spinner on the figure) were installed at 6,18 , 30 , and $42 \mathrm{~m}$ downwind from spray structure moving line on the top of one monofilament line station on the east side at the distance. Each rotary impactor was mounted a pair of $7.6 \mathrm{~cm}$ glass bars to capture fine droplets floating in the air.

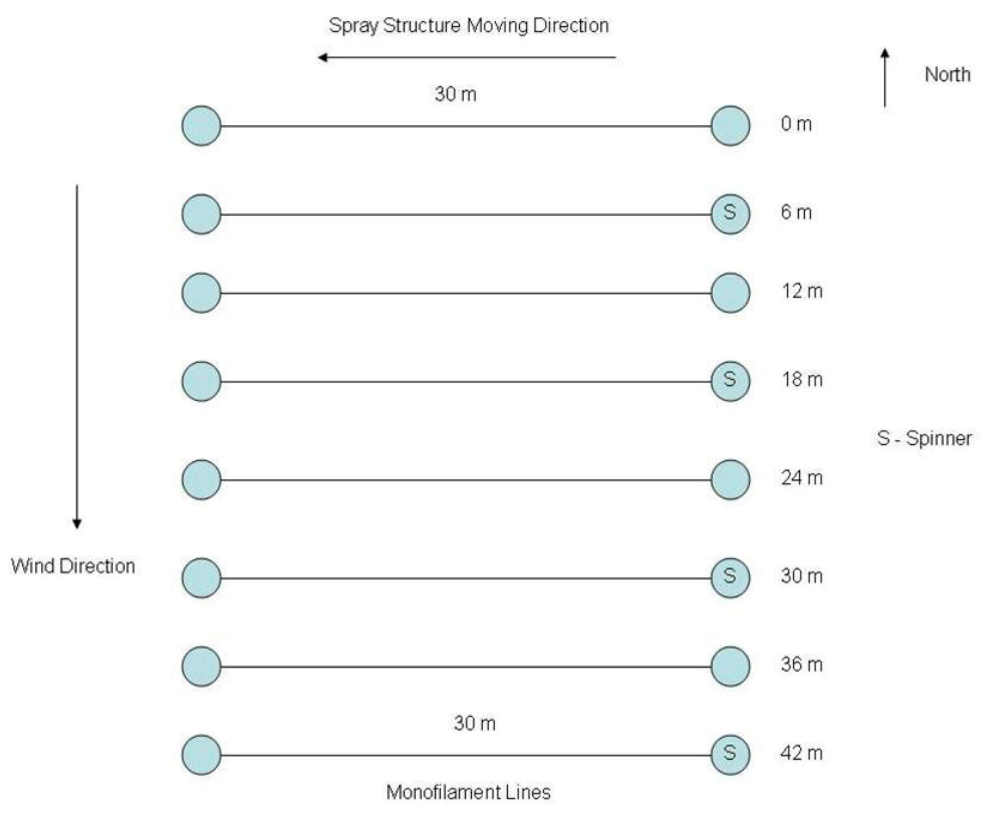

Figure 2. Field layout of the low-volume sprayer test 


\subsection{Field Evaluation Operation}

Eight passes were run for the test with two DC power voltages $\times$ two release heights $\times$ two replications. Sixty four PVC (Polyvinyl chloride) tube holders were used to hold the spray lines collected with 8 passes $\times 8$ lines. Thirty two labeled polyethylene bags were used to hold 32 pairs of acryl glass bars from rotary impactors with 8 passes $\times 4$ stations.

Meteorological data was measured using a Kestrel (Nielsen-Kellerman, Boothmyn, Pennsylvania, USA) 4000 Pocket Wind Meter $(0.4-60 \mathrm{~m} / \mathrm{s}$ with a resolution of $0.1 \mathrm{~m} / \mathrm{s}$ measured every second). Wind speed was logged starting with the spray application and continuing for 5 minutes. The data was logged using the devices internal memory and downloaded to a PC using the data connection interface. Wind speed data was averaged for each spray application and for the over study period. Overall study wind speed average was $1 \mathrm{~m} / \mathrm{s}$ with very little variation between applications.

\subsection{Field Sample Processing}

Sample processing was conducted by pipetting 10 and $30 \mathrm{ml}$ of $100 \%$ hexane wash into each polyethylene bag and each PVC line holder, respectively, agitating the sample bag and holder (approx. $15 \mathrm{sec}$ for acryl glass bars and $30 \mathrm{sec}$ for monofilament lines), and decanting $6 \mathrm{ml}$ into a cuvette. The dye concentration $(\mu \mathrm{g} / \mathrm{ml})$ of samples and tank mixture were measured using a spectrofluorometer (Shimadzu, Model RF5000U, Kyoto, Japan) with an excitation wavelength of $427 \mathrm{~nm}$ and an emission at $489 \mathrm{~nm}$. The minimum detection level for the dye and sampling technique was $0.07 \mathrm{ng} / \mathrm{ml}$. The sample concentration was multiplied by the wash volume and divided by the effective sample area to get $\mu 1$ of dye $/ \mathrm{cm}^{2}$.

\subsection{Statistical Analysis}

Statistical analysis was conducted to profile the spray deposition downwind under different power voltages, release heights, and downwind distance. For the analysis the SAS GLM procedure was run using SAS for Windows software (version 9.1.3) (SAS Institute Inc., Cary, NC, USA).

\section{Results and Discussion}

Tables 1, 2 and 3 show the statistical results of Uvitex concentration responses in hexane to power voltage, release height and downwind distance, respectively, from monofilament line measurement for spray deposition downwind using mean separation by Duncan grouping. The results illustrate that the two voltage levels of the DC power had significantly different impact on the spray deposition downwind, but the two release heights had no significant impact on the spray deposition. In average the pattern of the spray deposition downwind varied over the swath with the minimal value of $2.67 \times 10^{-5} \mu \mathrm{l} / \mathrm{cm}^{2}$ at $30 \mathrm{~m}$ downwind and the maximal value of $5.36 \times$ $10^{-5} \mu \mathrm{l} / \mathrm{cm}^{2}$ at $36 \mathrm{~m}$ downwind. This sprayer could deliver fair amount of liquid $40 \mathrm{~m}$ downwind in the $30 \mathrm{~m}$ swath with small droplet size (less than $50 \mu \mathrm{m}$ VMD) (Huang et al., 2009).

Table 1. Uvitex concentration response in hexane to power voltage from monofilament line measurement using mean separation by Duncan grouping* $(p=0.0079)$

\begin{tabular}{ll}
\hline Voltage $(\mathbf{V})$ & Uvitex Concentration $\left(\boldsymbol{\mu l} / \mathbf{c m}^{2}\right)$ \\
\hline $\mathbf{4}$ & $4.58 \times 10^{-5 \mathrm{a}}$ \\
$\mathbf{6}$ & $3.26 \times 10^{-5 b}$ \\
\hline me column the quantity is not significantly different from the other one with the same letter.
\end{tabular}

* In the same column the quantity is not significantly different from the other one with the same letter.

Table 2. Uvitex concentration response in hexane to release height from monofilament line measurement using mean separation by Duncan grouping $(p=0.1305)$

\begin{tabular}{ll}
\hline Release Height $(\mathbf{m})$ & Uvitex Concentration $\left(\boldsymbol{\mu l} / \mathbf{c m}^{2}\right)$ \\
\hline $\mathbf{3 . 7 6}$ & $3.56 \times 10^{-5 \mathrm{a}}$ \\
$\mathbf{6 . 1}$ & $4.29 \times 10^{-5 \mathrm{a}}$ \\
\hline
\end{tabular}


Table 3. Uvitex concentration response in hexane to downwind distance from monofilament line measurement using mean separation by Duncan grouping $(\mathrm{p}=0.0304)$

\begin{tabular}{ll}
\hline Distance (m) & Uvitex Concentration $\left(\boldsymbol{\mu l} / \mathbf{c m}^{\mathbf{2}}\right)$ \\
\hline $\mathbf{0}$ & $3.40 \times 10^{-5 \mathrm{ba}}$ \\
$\mathbf{6}$ & $3.15 \times 10^{-5 \mathrm{~b}}$ \\
$\mathbf{1 2}$ & $5.31 \times 10^{-5 \mathrm{a}}$ \\
$\mathbf{1 8}$ & $3.77 \times 10^{-5 \mathrm{ba}}$ \\
$\mathbf{2 4}$ & $3.06 \times 10^{-5 \mathrm{~b}}$ \\
$\mathbf{3 0}$ & $2.67 \times 10^{-5 \mathrm{~b}}$ \\
$\mathbf{3 6}$ & $5.36 \times 10^{-5 \mathrm{a}}$ \\
$\mathbf{4 2}$ & $4.67 \times 10^{-5 \mathrm{ba}}$ \\
\hline
\end{tabular}

Extractable Uvitex concentration from deposition on the rotary impactors is presented in response to power voltage, release height and downwind distance, respectively, in Tables 4, 5 and 6 . Using the filament technique a higher concentration was observed compared to the rotary impactor methodology as a result of the greater collection efficiency of the monofilament lines $(72 \%$ versus $15 \%$ for the monofilament versus the Hock rotary impactor over the sampling area (Fritz et al., 2011). Measuring downwind coverage only a two-fold difference in deposition was observed, however using the rotary impactor method a 30-fold difference in deposition was observed with limited statistical significance $(\mathrm{p}=0.1028)$. The monofilament line is less sensitive to large scale spray movement as a result of sampling across a 30-m swath, wheras the rotary impactor samples from a $7 \times 16$ $\mathrm{cm}$ window. The model is not as significant as the one for monofilament line measurement. The results illustrate that the two voltages of the DC power had no different impact on the spray deposition downwind, and the two release heights had no too. In average the pattern of the spray deposition downwind varied over the swath with the minimal value of $7.24 \times 10^{-6} \mu \mathrm{l} / \mathrm{cm}^{2}$ at the downwind distance $6 \mathrm{~m}$ and the maximal value of $2.78 \times 10^{-5}$ $\mu \mathrm{l} / \mathrm{cm}^{2}$ at the downwind distance $18 \mathrm{~m}$.

Table 4. Uvitex concentration response in hexane to power voltage from rotary impactor measurement using mean separation by Duncan grouping $(p=0.3053)$

\begin{tabular}{ll}
\hline Votage $(\mathrm{V})$ & Uvitex Concentration $\left(\mu \mathrm{l} / \mathbf{c m}^{2}\right)$ \\
\hline $\mathbf{4}$ & $2.44 \times 10^{-5 \mathrm{a}}$ \\
$\mathbf{6}$ & $1.77 \times 10^{-5 \mathrm{a}}$ \\
\hline
\end{tabular}

Table 5. Uvitex concentration response in hexane to release height from rotary impactor measurement using mean separation by Duncan grouping $(\mathrm{p}=0.2060)$

\begin{tabular}{ll}
\hline Release Height $(\mathbf{m})$ & Uvitex Concentration $\left(\boldsymbol{\mu l} / \mathbf{c m}^{2}\right)$ \\
\hline $\mathbf{3 . 7}$ & $2.52 \times 10^{-5 \mathrm{a}}$ \\
$\mathbf{6 . 1}$ & $1.69 \times 10^{-5 \mathrm{a}}$ \\
\hline
\end{tabular}

Table 6. Uvitex concentration response in hexane to downwind distance from rotary impactor measurement using mean separation by Duncan grouping $(\mathrm{p}=0.1028)$

\begin{tabular}{ll}
\hline Distance (m) & Uvitex Concentration $\left(\boldsymbol{\mu l} / \mathbf{c m}^{2}\right)$ \\
\hline $\mathbf{6}$ & $7.24 \times 10^{-6 \mathrm{~b}}$ \\
$\mathbf{1 8}$ & $2.78 \times 10^{-5 \mathrm{a}}$ \\
$\mathbf{3 0}$ & $2.19 \times 10^{-5 \mathrm{ba}}$ \\
$\mathbf{4 2}$ & $2.73 \times 10^{-5 \mathrm{a}}$ \\
\hline
\end{tabular}




\section{Conclusion}

The field study indicated that the UAV technology is effective for precision delivery of low-volume chemicals with the droplets less than $50 \mathrm{~mm}$ VMD to meet the requirement of vector control. The low-volume sprayer for the UAV helicopter was able to deliver liquid covering the 30-m swath, 42-m downwind. Deposition results from monofilament lines demonstrated that the spray coverage was sensitive to the power voltage but not release height. The deposition measurement from rotary impactor methodology needs to be refined to achieve higher resolution and lower variance. The method, system and analytical results from this study provide an extendable prototype that could be used in developing UAV aerial application systems for crop production management with higher target rate and larger VMD droplet size (300 $\mathrm{m}$ and higher).

\section{References}

Fritz, B. K., Hoffmann, W. C., Bonds, J. A. S., \& Farooq, M. (2011). Volumetric collection efficiency and droplet size accuracy of rotating impactors. Transactions of the ASABE, 54(1), 57-63. http://dx.doi.org/10.13031/2013.36253

Huang, Y., Hoffmann, W. C., Lan, Y., Wu, W., \& Fritz, B. K. (2009). Development of a Spray System for an Unmanned Aerial Vehicle Platform. Applied Engineering in Agriculture, 25(6), 803-809. http://dx.doi.org/10.13031/2013.29229

Huang, Y., Thomson, S. J., Hoffmann, W. C., Lan, Y., \& Fritz, B. K. (2013). Development and prospect of unmanned aerial vehicle technologies for agricultural production management. International Journal of Agricultural and Biological Engineering, 6(3), 1-10. http://dx.doi.org/10.3965/j.ijabe.20130603.001

Miller, J. W. (2005). Report on the Development and Operation of an UAV for an Experiment on Unmanned Application of Pesticides. Air Force Research Laboratory, United States Air Force.

Sato, A. (2003). The RMAX Helicopter UAV. Report of the Aeronautic Operations. Yamaha Motor CO., LTD., Shizuoka, Japan.

\section{Copyrights}

Copyright for this article is retained by the author(s), with first publication rights granted to the journal.

This is an open-access article distributed under the terms and conditions of the Creative Commons Attribution license (http://creativecommons.org/licenses/by/3.0/). 